\title{
Термодинамические ограничения и информачионные условия устойчивости, управляемости и робастности интеллектуального когнитивного управления
}

\author{
С.В. Ульянов 1, 2, д.фр.-м.н., npoфpeccop, ulyanovsv46_46@mail.ru \\ A.A. Шевченко ${ }^{1}$, аспирант, allabard@yandex.ru \\ A.B. Шевченко ${ }^{1}$, аспирант, sh3vchenkoav@yandex.ru \\ О.Ю. Тятюшкина ${ }^{1}$, к.m.н., доиент, tyatyushkina@mail.ru \\ ${ }^{1}$ Государственный университет "Дубна" - Институт системного анализа \\ и управления, г. Дубна, 141980, Россия \\ 2 Объединенный институт ядерных исследований - лаборатория \\ информаиионных технологий, г. Дубна, 141980, Россия
}

В данной статье рассмотрены информационные и физические (энтропийные и энергетические) закономерности, а также особенности модели квантового сильного искусственного вычислительного интеллекта в виде самоорганизующейся интеллектуальной системы управления. Модель основана на принципах минимальной информационной энтропии (в «интеллектуальном» пространстве состояний сигналов управления) и минимальной обобщенной термодинамической меры производства энтропии в единой системе «объект управления + интеллектуальный когнитивный регулятор». Основным результатом применения процесса самоорганизации является гарантированная возможность достижения необходимого уровня надежности и гибкости (адаптивности) воспроизводимой структуры когнитивной интеллектуальной системы управления.

В работе кратко рассмотрены основные физические принципы процессов управления, позволяющие устанавливать взаимосвязь между качественными характеристиками динамического поведения объекта управления и исполнительным устройством системы автоматического управления: устойчивостью, управляемостью и робастностью управления. Для достижения этой цели используются информационный и термодинамический подходы, объединяющие однородным условием критерии динамической устойчивости (функция Ляпунова), управляемости и робастности.

Приведены соотношения между количеством совершенной работы, информации и извлекаемой свободной энергии, которые подтверждают возможность повышения робастности интеллектуальной системы управления за счет производства энтропии когнитивного регулятора, уменьшающего потери полезного ресурса объекта управления. В свою очередь, производство негэнтропии когнитивного регулятора снижает требования к минимуму исходной информации для достижения робастности. На основе извлекаемой информации из баз знаний когнитивного регулятора возможно получить дополнительный ресурс для совершения полезной работы, эквивалентный целенаправленному действию на объект управления, гарантируя достижение цели управления.

Ключевые слова: квантовый алгоритм самоорганизаций, неточные знания, термодинамика информационных процессов, когнитивные системы управления.

Стремительное развитие квантовой релятивистской механики и термодинамики заложило фундамент для нового поколения квантовых сквозных информационных технологий, что послужило основой развития квантовых инженерии (например, аппаратная реализация кван-тового компьютера), теории информации, программной инженерии, вычислений, криптографии, алгоритмов и программирования.

Информационно-термодинамический закон распределения качеств управления, таких как устойчивость, управляемость и робаст- ность, позволяет проектировать встраиваемые самоорганизующиеся интеллектуальные когнитивные системы управления, повышая эффективность технологических процессов и исполнительных устройств без изменения конструктивных решений (принцип неразрушения исполнительского уровня).

Совместное включение в контур структуры системы управления когнитивного и интеллектуального (нечеткого) регуляторов позволяет использовать когнитивный потенциал человека-оператора и применять принцип компенсации ошибки управления от не- 
штатной ситуации управления соответствующей структурой регуляторов. При этом базы знаний (БЗ) регуляторов возможно спроектировать на основе квантового нечеткого вывода, реализующего квантовый алгоритм самоорганизации неточных Б3.

Более того, природа и сущность самого понятия «информация» могут рассматриваться как материальный физический объект, дающий возможность совершать полезную работу. Это позволяет установить механизм и возможности такой работы, не нарушая второго закона термодинамики, за счет корректных информационных моделей самого второго закона. В результате количество информации, извлеченное на квантовом уровне из когнитивных процессов, позволяет совершать на макроуровне количество полезной работы, большее по сравнению с работой, затраченной на ее извлечение на микроуровне, без нарушения второго закона термодинамики.

При проектировании систем управления приходится учитывать не только известные в теории управления ограничения, но и новые физические явления и эффекты. Для практической реализации квантового подхода в процессе проектирования эффективных алгоритмов принятия решений и систем управления в условиях ограничений количества доступной информации требуется повышение уровня интеллектуальности, позволяющее включить в формируемые законы управления квантовые эффекты и явления.

\section{Термодинамическое распределение и соотношение критериев устойчивости, управляемости и робастности}

Для достижения необходимого уровня робастности и повышения интеллектуальности системы управления важно учитывать термодинамическое распределение, а также соотношение критериев устойчивости, управляемости и робастности интеллектуального управления. Рассмотрим динамический объект управления (ОУ), описываемый (в общем виде) уравнением

$$
\frac{d q}{d t}=\varphi(q, t, S(t), u(t)),
$$

где $q$ - вектор обобщенных координат, описывающий динамическое поведение ОУ; $u$ управляющая сила (выход исполнительного устройства системы автоматического управления (САУ)); $t$ - время; $S(t)$ - обобщенное производство энтропии системы «ОУ + регулятор».
Необходимые и достаточные условия асимптотической устойчивости динамической системы, описываемой уравнением (1), определяются физическими ограничениями на вид функции Ляпунова, которая имеет два важных свойства: это строго положительная функция от обобщенных координат, то есть $V>0$ (условие 1), полная производная по времени от функции Ляпунова является неположительной функцией, $\frac{d V}{d t} \leq 0$ (условие 2).

Согласно условиям 1 и 2 в качестве обобщенной функции Ляпунова выберем следующую функцию:

$$
V=\frac{1}{2} \sum_{i=1}^{n} q_{i}^{2}+\frac{1}{2} S^{2},
$$

где $S=S_{p}-S_{c}-$ производство энтропии в открытой системе «ОУ + регулятор»; $S_{p}$ - производство энтропии в ОУ; $S_{c}-$ производство энтропии в регуляторе (в исполнительном устройстве САУ).

Отметим, что система (1) является открытой динамической системой в термодинамическом смысле, а основная задача регулятора - снижение энтропии динамического поведения ОУ. Поэтому стоит знак вычитания энтропии, производимой регулятором (а не плюс, как в случае замкнутой термодинамической системы), что приводит к понятию негэнтропии Бриллюэна. Введение энтропийных характеристик в (2) возможно в силу скалярного свойства энтропии как функции времени, $S(t)$. Первое условие для функции Ляпунова (2) выполняется автоматически.

Потребуем выполнения второго условия: $\frac{d V}{d t} \leq 0$. В этом случае полная производная от функции Ляпунова, описанной в (2), имеет вид

$$
\begin{aligned}
& \frac{d V}{d t}=\frac{1}{2} \sum 2 \dot{q}_{i} q_{i}+\frac{1}{2} 2 S \dot{S}=\sum_{i=1}^{n} \dot{q}_{i} q_{i}+S \dot{S}= \\
& =\sum_{i=1}^{n} q_{i} \varphi(q, t, S(t), u)+\left(S_{p}-S_{c}\right)\left(\dot{S}_{p}-\dot{S}_{c}\right) .
\end{aligned}
$$

Таким образом, учитывая соотношение (1), имеем

$$
\begin{gathered}
\frac{d V}{d t}=\underbrace{\sum_{i=1}^{n} q_{i} \varphi(q, t, S(t), u)}_{\text {устойчивость }}+ \\
+\underbrace{\left(S_{p}-S_{c}\right) \cdot\left(\dot{S}_{p}-\dot{S}_{c}\right)}_{\text {робастность }} \leq 0 .
\end{gathered}
$$

На рисунке 1 показана взаимосвязь между функцией Ляпунова и производством энтропии в ОУ и в САУ. 


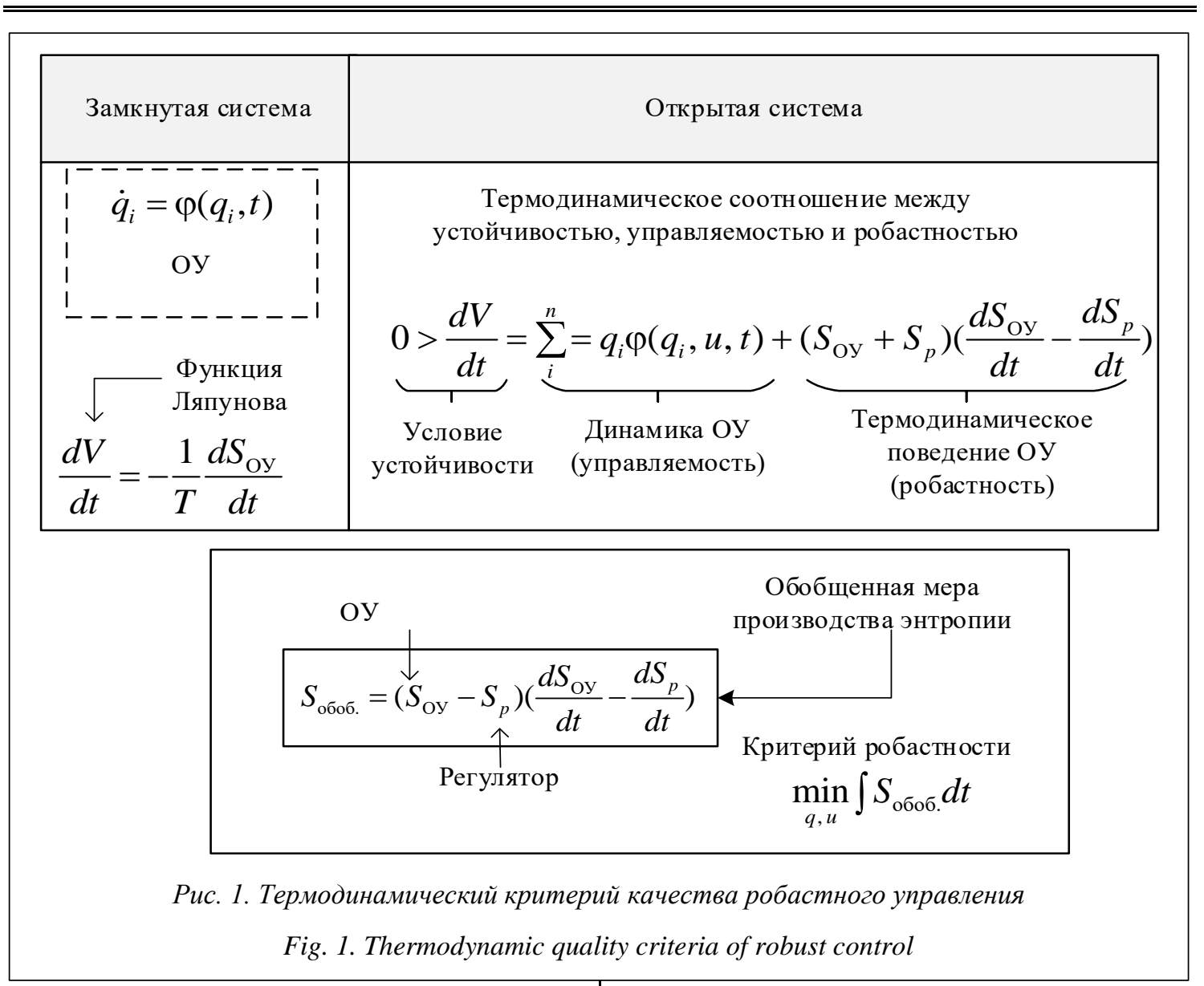

Далее необходимо учитывать информационные условия и ограничения на процессы когнитивного управления. Уравнение (3) описывает физический закон качества управления с позиции ограничений и требований второго закона термодинамики и объединяет в аналитической форме различные меры качества управления типа устойчивость, управляемость и робастность, поддерживающие требуемую надежность и точность управления.

Следовательно, взаимосвязь между устойчивостью по Ляпунову и робастностью, описанная уравнением (3), является основным физическим законом для проектирования САУ. Этот закон - основа для прикладной технологии проектирования БЗ робастных интеллектуальных систем управления (ИСУ) (с различными уровнями интеллектуальности $[1,2])$ с использованием технологий мягких вычислений.

Практическое применение физического закона процессов управления (3) к традиционным задачам, таким как оценка точности (грубости) линеаризации моделей ОУ, наблюдаемости параметров процессов управ- ления и др., рассмотрено в [1]. Следует отметить, что член $\sum_{i} q_{i} \dot{q}_{i}$ характеризует дополнительную возможность работы с физической моделью ОУ без применения математической модели, используя непосредственно измерение динамического поведения ОУ. В этом случае имеем обобщение модели черного ящика ОУ.

Уравнение (3) связывает в аналитической форме такие качественные понятия теории управления $u$, как устойчивость, управляемость и робастность, на основе понятия энтропии феноменологической термодинамики с учетом совершения полезной работы регулятора.

Данный подход позволяет, как отмечалось ранее, найти такое управление $u$, которое имеет необходимое распределение между уровнями устойчивости, управляемости и робастности и позволяет достигать цели управления в нештатных ситуациях с минимальным расходом полезного ресурса за счет применения в качестве функции пригодности в генетическом алгоритме минимума производства обобщенной энтропии, входящей в правую часть (3). 
Пример. Термодинамическая интерпретация информационной энтропии Шеннона эквивалентность мер энтропий. Для оценки информационных возможностей интеллектуального когнитивного регулятора рассмотрим важную связь между термодинамической $S$ и информационной $H$ энтропиями. Рассмотрим для переменной $X$ так называемую зетафункцию Хасcе-Вейля (Hasse-Weil $(H W)$ ) следующего вида: $Z(X, t)=\prod_{r \geq 1}\left(1-t^{r}\right)^{-a_{r}}$. При замене переменных $t=q^{-s}$ можно обозначить зета-функцию Хассе-Вейля через $Z\left(X, q^{-s}\right)$. Согласно $[3,4]$, информационная энтропия Шеннона $H(X, s)$ для вероятностного распределения $P(n, x)=\frac{t^{n}}{Z^{H W}(X, t)}, n \in X$, имеет вид $H(X, t)=-\sum_{n, x} P(n, x) \log P(n, x)$. Оказалось, что между информационной энтропией Шеннона и зета-функцией Хассе-Вейля $Z^{H W}\left(X, q^{-s}\right)$ имеется следующее соотношение: $H(X, s)=\left(1-s \frac{d}{d s}\right) Z^{H W}\left(X, q^{-s}\right)$ (см. [4], 6.2.1. Lemma). Для свободной энергии $F$ физической системы $F=-\log Z(\beta), \beta=\frac{1}{T}>0$, где $T$ - температура, $Z(\beta)$ - так называемый частный случай функции $Z^{H W}\left(X, q^{-s}\right)$, приведенная лемма показывает, что информационная энтропия Шеннона $H(X, s)$ полностью согласуется c термодинамической энтропией $S=\left(1-\beta \frac{\partial}{\partial \beta}\right) \log Z(\beta)$. В этом случае зета-функция Хассе-Вейля $Z^{H W}\left(X, q^{-s}\right)$ идентифицируется частной функцией $Z(\beta)$ физической системы с гамильтонианом $H$ таким образом, что выполняется соотношение $\operatorname{Tr}\left(e^{-\beta H}\right)=$ $=Z^{H W}\left(X, q^{-\beta}\right)$. Тогда выражение для термодинамической энтропии совпадает с соотношением для информационной энтропии Шеннона для распределения вероятностей вида $P(n)=\frac{e^{-\beta \lambda_{n}}}{Z(\beta)}$ при $Z(\beta)=\operatorname{Tr}\left(e^{-\beta H}\right)$ и $\operatorname{Spec}(H)=$ $=\lambda_{n}$, так как имеем

$S=-\sum_{n} P(n) \log P(n)=\sum_{n} P(n) \log Z(\beta)-\beta \sum_{n} P(n) \lambda_{n}=$ $=\left(\left[\sum_{n} P(n)=1\right] \log Z(\beta)-\beta\left[\sum_{n} P(n) \lambda_{n}=\frac{\partial}{\partial \beta} \log Z(\beta)\right]\right)=$ $=\left(1-\beta \frac{\partial}{\partial \beta}\right) \log Z(\beta)$,

где $\sum_{n} P(n) \lambda_{n}=\frac{\partial}{\partial \beta} \log Z(\beta)$.
Таким образом, в результате вычислений имеем прямое доказательство эквивалентности мер энтропий:

$$
S=\left(1-\beta \frac{\partial}{\partial \beta}\right) \log Z(\beta)=-\sum_{n} P(n) \log P(n)=H .
$$

Рассмотрим теперь (3) с учетом приведенной связи термодинамической энтропии с информационной энтропией Шеннона. Определения термодинамической энтропии $S$ и информационной $H$ связаны соотношением фон Неймана [1] в виде

$$
S=k H=-k \sum_{i} p_{i} \ln p_{i},
$$

где $k \approx 1,38 \cdot 10^{-23}$ Дж/К - постоянная Больцмана. Подставим в уравнение (3) вместо $S(t)$ информационную энтропию Шеннона $H$. В результате получим

$$
\begin{aligned}
& \underbrace{\frac{d V}{d t}}_{\text {устойчивость }}=\underbrace{\sum_{i=1}^{n} q_{i} \varphi\left(q, t, k\left(H_{p}-H_{c}\right), u\right)}_{\text {управляемость }}+ \\
& +\underbrace{k \underbrace{\left(H_{p}-H_{c}\right)\left(H_{p}-\dot{H}_{c}\right)}_{p} \leq 0 .}_{\text {робастность }}
\end{aligned}
$$

Таким образом, уравнение (4) на основе информационной энтропии Шеннона связывает устойчивость, управляемость и робастность, а также позволяет определять управление $u$ для гарантированного достижения цели управления в нештатных ситуациях с требованием минимального количества информации о внешней среде и о состоянии объекта управления. Следовательно, уравнения (3) и (4) включают в себя перечисленные возможности применения физической или информационной энтропии проектируемого управления $u$ в качестве полезного ресурса для достижения глобальной робастности ИСУ.

\section{Информационно-термодинамический закон квантовой самоорганизации интеллектуального управления}

Как отмечалось, уравнения (3) и (4) составляют систему уравнений, определяющую такое управление $u$, которое гарантирует достижение цели управления в нештатных ситуациях с минимальным расходом полезного ресурса и минимально требуемой исходной информацией. Рассмотрим некоторые особенности связи понятий количества информации и физической энтропии в свете приведенных уравнений (3) и (4). Так, определение физической энтропии дает возможность количественной формулировки второго закона термодинамики, который в изолированной 
системе запрещает процессы, сопровождающиеся уменьшением энтропии. Достаточно обратить внимание на формулировку связи функции Ляпунова и производства энтропии для замкнутой в термодинамическом смысле системы на рисунке 1 в виде $\frac{d S}{d t}=-\frac{1}{T} \frac{d V}{d t}$.

Из определения функции Ляпунова динамическая устойчивость эволюции системы $V>0, \frac{d V}{d t} \leq 0$, что приводит к условию второго закона термодинамики $d S \geq 0$ для замкнутой системы в термодинамическом смысле.

При этом, однако, если возможен приток информации $d I$ о системе, то есть если физическая система является изолированной только в тепловом, но не в информационном отношении, то указанный закон следует обобщить [5, 6], заменив неравенство $d S \geq 0$ неравенством $d S+d I \geq 0$. Поэтому, если имеется приток информации, можно тепловую энергию системы превратить в механическую без помощи холодильника.

Таким образом, можно построить вечный двигатель второго рода, питающийся информацией. Особо следует подчеркнуть, что информационная форма обобщения второго закона термодинамики не отменяет его первоначальной формулировки. Отсюда следует заключение о необходимости энергетических затрат при измерении координат физической системы и записи этой информации. Если система находится при температуре $T$, то для получения и записи количества информации $d I$ о ней необходимо потратить как минимум $T d I$ энергии. В противном случае соединение автоматического измерителя и информационного преобразователя тепловой энергии в механическую дало бы вечный двигатель второго рода. Вывод о необходимости минимальных энергетических затрат распространяется также на физические каналы с шумом, соответствующим заданной температуре $T$.

Таким образом, второй закон термодинамики накладывает некоторые ограничения на возможности физической реализации информационных систем, в частности, автоматовизмерителей и каналов передачи информации. Максимальное количество тепловой энергии, переходящее в работу, равно произведению абсолютной температуры на количество приходящей информации Больцмана. Приток информации о физической системе позволяет переводить тепловую энергию в работу без передачи части тепловой энергии холодильнику. Информация в этом смысле является также физическим процессом, способным создавать ресурс для совершения дополнительной полезной работы.

Пример. Демон Максвелла. Возможность распространения описанного обобщения второго закона термодинамики на случай систем с притоком информации возникла давно в связи с обсуждением демона Максвелла. Последний, открывая и закрывая дверцу в стенке между двумя сосудами, в зависимости от скорости, с которой молекула подлетает к дверце, может создать разность температур или давлений, не совершая работы и нарушая второй закон термодинамики. В этом случае демону необходим приток информации. Пределы нарушения демоном второго закона термодинамики ограничены величиной приходящей информации. Это можно утверждать не только качественно, но и количественно согласно закону $d S+d I \geq 0$, то есть в виде точного количественного закона. Обозначим через $d Q$ количество теплоты, пришедшее из термостата. Запишем изменение энтропии термостата в виде $d S_{T}=-\frac{d Q}{T}$. По первому закону термодинамики $d A=d Q-d U$, где $U=M[E]-$ внутренняя энергия системы, связанная со свободной энергией $F$ известным соотношением $U=F+T S_{x}$. Дифференцируя последнее соотношение, имеем $d F=d U-$ $-T d S_{x}$. Второй закон термодинамики имеет в этом случае вид $d S_{T}+d S_{x} \geq 0, T S_{x}-d Q \geq 0$, что эквивалентно соотношению $d A \leq-d F$. Указанный энергетический переход совершается без изменения температуры системы. Это имеет место вследствие притока тепловой энергии из термостата, контакт с которой не должен прерываться. Тогда источником уходящей из системы механической энергии будет тепловая энергия термостата, превращающаяся в механическую работу.

Таким образом, если физическая система изолирована в тепловом отношении и о ней имеется количество информации $I$, то в системе возможны только такие процессы, для которых изменение суммарной энтропии превосходит $(-I): \Delta S \geq-I$. При этом нижняя граница физически достижима. Под тепловой изоляцией подразумевается то, что тепловая энергия, которую при указанных процессах можно превратить в работу, берется из самой 
системы, то есть термостат включен в данную систему.

Второй закон термодинамики, как известно, нарушается для процессов, связанных с тепловыми флуктуациями, то есть является асимптотическим и не вполне точным. Можно дать уточненную формулировку: в теплоизолированной системе не могут происходить процессы, для которых приращение энтропии $\Delta S \ll-1$. Если брать энтропию в термодинамических единицах, то вместо 1 следует поставить постоянную Больцмана $k$. Тогда последнее соотношение примет вид $\Delta S \ll-k$. Соответственно, в уточненной формулировке запрещены процессы, для которых $\Delta S+I \ll-1$ или $\Delta S_{\text {физ. }}+k I \ll-k$. Член с количеством информации $I$ здесь существенен, если $I \gg 1[7,8]$.

Дальнейшие исследования позволили уточнить роль квантового второго закона термодинамики в квантовой теории информации, взаимосвязь понятий работы диссипативных процессов с понятиями относительного количества информации Фишера [9-12], а также связи понятия производимой работы с количеством приходящей информации [13-16].

Таким образом, уравнения (3) и (4) включают в себя перечисленные возможности применения физической или информационной энтропии в качестве полезного ресурса для достижения глобальной робастности ИСУ.

Пример. Работа диссипативных сил (как индикатор доступа к оченке затраченной системой работы). Определяется в терминах меры свободной энергии $\Delta F$ (отличие от равновесного состояния) как $W_{\text {дисс. }}(\Gamma, \lambda)=$ $=W(\Gamma, \lambda)-\Delta F$, где $\lambda-$ параметр протокола; $W(\Gamma, \lambda)$ означает усредненную работу, произведенную системой при воздействии внешних сил, и является функцией положения в фазовом пространстве Г. Работа диссипативных сил представляет собой полное изменение производства энтропии системы как результат переноса потока тепла от действия внешних сил. Свободная энергия как мера различия конечных состояний системы называется также обратимой работой. Одновременно существует эквивалентное описание процессов с точки зрения статистического ансамбля реализации рассматриваемого процесса как последовательности бесконечно воспроизводимых траекторий данного процесса. Поэтому можно рассматривать работу диссипативных сил как усредненную величину $\left\langle W_{\text {дисс. }}(\Gamma, \lambda)\right\rangle$. При этом между величиной $W_{\text {дисс. }}(\Gamma, \lambda)$ и информационным расхождением Кульбака-Лейблера существует следующая связь: $\frac{\left\langle W_{\text {дисс. }}\right\rangle}{k T}=S_{K L}\left(P_{F} \mid P_{B}\right)$, где $P_{F}$ и $P_{B}$ - распределения плотности вероятностей в фазовом пространстве обратимых во времени процессов для данного момента времени. Таким образом, левая часть указанного соотношения - тепловая энергия с постоянной Больцмана для температуры $T$, а правая - информационная величина. Кроме того, если для распределений $f$ и $g$ ввести оценку количества относительной информации Фишера в виде $S_{R F I}(f \mid g)=\int f\left|\nabla_{\Gamma}\left(\ln \frac{f}{g}\right)\right|^{2} d \Gamma$, то имеем соотношение $S_{R F I}\left(P_{F} \mid P_{B}\right)=\beta^{2}\left\langle\left|\nabla_{\Gamma} W_{\text {дисс. }}(\Gamma)\right|^{2}\right\rangle_{P_{F}}$.

Усредненный градиент работы диссипативных сил, производимой переходным процессом от состояния равновесия, эквивалентен расстоянию между прямым и обратным распределениями в фазовом пространстве, которое определяется количеством относительной информации Фишера.

Пример. Квантовая система с диссипаичей. В уравнениях движения квантовых систем диссипация описывается феноменологически логарифмической нелинейностью и феноменологической постоянной $\gamma$. В общем случае эволюция диссипативных процессов рассматриваемой динамической системы с гамильтонианом $H$ описывается матрицей плотности $\varrho$ в виде уравнения

$$
\dot{\varrho}=\frac{1}{i \hbar}[H, \varrho]+\gamma D(\varrho),
$$

где $D(\varrho)$ - диссипативный оператор. В (5) предполагается в дальнейшем, что $\varrho(t)$ зависит только от настоящего времени $t$, то есть процесс без истории, $t^{\prime}<t$. При $\gamma=0$ имеем уравнение фон Неймана

$$
\dot{\varrho}=\frac{1}{i \hbar}[H, \varrho]
$$

решение которого сохраняет инвариант вида $\langle S\rangle=\operatorname{Tr} \varrho S$ и $S=-\ln \varrho$. Следует подчеркнуть, что решение уравнения (5) сохраняет инвариант как для чистых $\left(\varrho^{2}=\varrho\right)$, так и для смешанных $\left(\varrho^{2} \neq \varrho\right)$ состояний.

Для оператора $\varrho$ вводятся следующие аксиомы: 1) Tr $\varrho=1$; 2) $\varrho(t)$ - эрмитов положительный оператор; 3) для двух несвязанных и независимых систем $H=H_{1}+H_{2}$ имеем 
$\varrho(t)=\varrho_{1} \otimes \varrho_{2}$. По аналогии с введенными аксиомами для оператора $D(\varrho)$ имеем:

1) $\operatorname{Tr} D(\varrho)=0$

2) $D(\varrho)^{\dagger}=D(\varrho)$;

3) $D\left(\varrho_{1} \otimes \varrho_{2}\right)=D\left(\varrho_{1}\right) \otimes \varrho_{2}+\varrho_{1} \otimes D\left(\varrho_{2}\right)$.

Если ввести обозначение $\tilde{D}$ и определить оператор $\tilde{D}$ как $D(\varrho)=\tilde{D(}(\varrho) \varrho$, то получим

$\tilde{D}\left(\varrho_{1} \otimes \varrho_{2}\right)\left(\varrho_{1} \otimes \varrho_{2}\right)=$

$=\tilde{D}\left(\varrho_{1}\right) \varrho_{1} \otimes \varrho_{2}+\varrho_{1} \otimes \tilde{D}\left(\varrho_{2}\right) \varrho_{2}$.

Выполняется следующее соотношение:

$\left\langle\tilde{D}\left(\varrho_{1} \otimes \varrho_{2}\right)\right\rangle=\left\langle\tilde{D}\left(\varrho_{1}\right)\right\rangle+\left\langle\tilde{D}\left(\varrho_{2}\right)\right\rangle$,

то есть оператор $\tilde{D}(\varrho)$ аддитивен.

Решение функционального уравнения (7) имеет вид

$$
\tilde{D_{1}}(\varrho)=-\ln \varrho=S, \tilde{D_{2}}(\varrho)=\langle-\ln \varrho\rangle \varrho
$$

или комбинацию из двух решений в (9).

Для выполнения аксиомы $\operatorname{Tr} D(\varrho)=0$ (сохранение вероятности) следует определить оператор $\tilde{D(\varrho)}$ в виде

$$
\tilde{D}(\varrho)=S-\langle S\rangle \|
$$

или

$$
\tilde{D}(\varrho)=(\langle S-\langle S\rangle \|\rangle) \varrho .
$$

Оказалось, что формы (10) и (11) не являются единственными.

В частности,

$$
D_{A}(\varrho)=\frac{1}{2}[A, \varrho]_{+}-\langle A\rangle \varrho,
$$

где $A$ является эрмитовым оператором; $[\cdot, \cdot \cdot]$ - антикоммутатор; $A=A_{1}+A_{2}$ удовлетворяет соотношению (7). Из (5) следует обобщение (6) с учетом, например, (11) в виде

$$
\dot{\varrho}=\frac{1}{i \hbar}[H, \varrho]+\gamma(\langle S-\langle S\rangle \amalg\rangle) \varrho .
$$

Таким образом, получено обобщение результатов на квантовые динамические системы и критерии физической реализуемости соответствующих математических моделей [17]. Следовательно, термодинамический критерий физической реализуемости выполняется - исследуемая математическая модель корректна.

\section{Информационные функции Ляпунова и квантовая функция Фишера}

В квантовой механике гильбертово пространство состояний связано с геометрией пространств, в которых введены меры различимости состояний и существует возможность определения чистых и смешанных состояний.

Метрика данной различимости связана естественным образом с представлением длины траектории вычисления квантового алгоритма, и естественным вопросом явля- ется выявление отношения между данной длиной и вычислительной сложностью программы. В этом случае траектория в гильбертовом пространстве, определяемая квантовым алгоритмом, является геодезической в метрике Фубини-Штади (Fubiny-Study).

Пример. Квантовые геометрические аналоги классических моделей кривизны в информационной геометрии искривленных пространственно-временных континуумов. Рассмотрим в качестве примера факты геометрии пространства квантовых состояний. Расстояние между двумя квантовыми состояниями $\left|\psi_{1}\right\rangle$ и $\left|\psi_{2}\right\rangle$ можно определить различными способами. Например, широко применяемые меры расстояния $F S$ и Wootters $(W)$ определяются соответственно в следующем виде: $d^{(F S)}\left(\left|\psi_{1}\right\rangle,\left|\psi_{2}\right\rangle\right)=\gamma\left(\sqrt{1-\left|\left\langle\psi_{1} \mid \psi_{2}\right\rangle\right|^{2}}\right)$ и $d^{(W)}\left(\left|\psi_{1}\right\rangle,\left|\psi_{2}\right\rangle\right)=\arccos \gamma\left|\left\langle\psi_{1} \mid \psi_{2}\right\rangle\right|$,

где $\gamma$ - постоянная величина. Несмотря на внешнее различие, данные меры расстояния эквивалентно определяют расстояние между квантовыми состояниями. Так, для близких состояний, когда $\left|\left\langle\psi_{1} \mid \psi_{2}\right\rangle\right|^{2}=1-\delta^{2}$, где $\delta$ - малая величина, имеем соотношение $d^{(F S)}=$ $=d^{(W)}=\gamma \delta$. Подобным образом определяются другие меры расстояний. Аналогично данному результату элементы длины для семейства (множества) векторов квантовых состояний $\left|\psi\left(\xi^{1}, \xi^{2}, \ldots, \xi^{k}\right)\right\rangle$ параметризованных $k$ параметрами $\xi^{1}, \xi^{2}, \ldots, \xi^{k}$, определенные на заданных метриках расстояния, эквивалентны для различных определений расстояния $d s^{2}=g_{i j} d \xi^{i} d \xi^{j}$ с метрическим тензором

$$
g_{i j}=\gamma^{2} \operatorname{Re}\left(\left\langle\psi_{i} \mid \psi_{j}\right\rangle-\left\langle\psi_{i} \mid \psi\right\rangle\left\langle\psi \mid \psi_{j}\right\rangle\right),
$$

где $\left.\left.\left|\psi_{i}\right\rangle=\frac{\partial}{\partial \xi^{i}} \| \psi\left(\xi^{1}, \xi^{2}, \ldots, \xi^{k}\right)\right\rangle\right\rangle$.

Приведенная форма метрики расстояния применяется на практике многими исследователями, и обычно принимается $\gamma=2$. Тогда для двумерного случая $g_{i j}$ является метрическим тензором сферы с единичным радиусом (сфера Блоха).

При рассмотрении эволюции квантового состояния, описываемой уравнением Шредингера, вводится понятие скорости квантовой эволюции $\vartheta=\frac{d s}{d t}=\frac{\gamma}{\hbar} \sqrt{\left\langle(\Delta \mathrm{H})^{2}\right\rangle}, \quad$ где $\Delta H=H-\langle H\rangle$. Рассмотрим теперь определение геодезической кривой на пространстве векторов квантовых состояний. Геодезическую линию (однопараметрическое множе- 
ство векторов квантовых состояний), соединяющую два вектора состояний $\left|\psi_{0}\right\rangle$ и $\left|\psi_{1}\right\rangle$, можно определить как линейную комбинацию (аналог суперпозиции):

$$
|\psi(\xi)\rangle=C\left[(1-\xi)\left|\psi_{0}\right\rangle+\xi\left|\psi_{1}\right\rangle e^{i \phi}\right],
$$

где $\xi$ - параметр, принимающий значения от 0 до 1.

Фазовый множитель $e^{i \phi}$ выбирается следующим образом. Векторы $\left|\psi_{0}\right\rangle$ и $\left|\psi_{0}\right\rangle e^{i \phi} 0$ определяют эквивалентные квантовые состояния. Поэтому требуется выполнение условия эквивалентности геодезических линий, определенных между состояниями $\left|\psi_{0}\right\rangle$ и $\left|\psi_{1}\right\rangle$ и состояниями $\left|\psi_{0}\right\rangle e^{i \phi_{0}}$ и $\left|\psi_{1}\right\rangle e^{i \phi_{1}}$. Данное условие выполняется, если выбрать $e^{i \phi}=\frac{\left\langle\psi_{1} \mid \psi_{0}\right\rangle}{\left|\left\langle\psi_{1} \mid \psi_{0}\right\rangle\right|}$.

Условие нормализации для параметра $C$ определяется из выражения внутреннего произведения $(\langle\psi(\xi) \mid \psi(\xi)\rangle=1$ и имеет следующий вид: $C=\frac{1}{\sqrt{1-2 \xi(1-\xi)\left(1-\left|\left\langle\psi_{1} \mid \psi_{0}\right\rangle\right|\right)}}$.

Отметим, что геодезическая линия (14) является множеством состояний и существует много способов ее параметризации. В геометрии квантовых состояний показано, что длина кривой в квантовом пространстве состояний не зависит от пути ее параметризации $[18,19]$.

Для вычисления длины геодезической линии удобно представлять определяющее ее уравнение в виде

$|\psi(\xi)\rangle=C\left[\sin \left(\frac{1}{2} \theta\right)\left|\psi_{0}\right\rangle+\cos \left(\frac{1}{2} \theta\right)\left|\psi_{1}\right\rangle e^{i \phi}\right]$,

где новый параметр $0 \leq \theta \leq \pi$ и константа нормализации $C=\frac{1}{\sqrt{1+\left|\left\langle\psi_{1} \mid \psi_{0}\right\rangle\right| \sin \theta}}$.

Подчеркнем, что (14) и (15) адекватно описывают однопараметрическое семейство векторов квантовых состояний в виде геодезических линий. Используя определение метрики как $g_{i j}=\gamma^{2} \operatorname{Re}\left(\left\langle\psi_{i} \mid \psi_{j}\right\rangle-\left\langle\psi_{i} \mid \psi\right\rangle\left\langle\psi \mid \psi_{j}\right\rangle\right)$ для однопараметрического множества состояний (15), получим $d s=\frac{\gamma}{2} \frac{\sqrt{1-\left|\left\langle\psi_{1} \mid \psi_{0}\right\rangle\right|^{2}}}{\left(1+\left|\left\langle\psi_{1} \mid \psi_{0}\right\rangle\right| \sin \theta\right)}$. Тогда длина геодезической линии, соединяющей состояния $\left|\psi_{0}\right\rangle$ и $\left|\psi_{1}\right\rangle$, определяется в виде

$$
S=\int d s=\gamma \arccos \left|\left\langle\psi_{1} \mid \psi_{0}\right\rangle\right| .
$$

Таким образом, выражение (16) для длины геодезической линии совпадает с выраже- нием метрики Wootters расстояния между векторами квантовых состояний. Аналогично можно вычислить длину кривой (14), соединяющей состояния $\left|\psi_{0}\right\rangle$ и $\left|\psi_{1}\right\rangle$, для определенной фазы $\phi$. Тогда геодезическая линия определяется как кривая минимальной длины на семействе кривых. Минимальная длина достигается при установленном выше условии $e^{i \phi}=\frac{\left\langle\psi_{1} \mid \psi_{0}\right\rangle}{\left|\left\langle\psi_{1} \mid \psi_{0}\right\rangle\right|}$ и эквивалентна метрике расстояния Wootters. Рассмотрим теперь другой геометрический фактор - кривизну пространства и его квантовый аналог в пространстве векторов квантовых состояний.

Кривизна. Вектор состояния квантовой эволюции от одного параметра, такого как время $t$, определим в виде однопараметрического множества векторов состояний $|\psi(t)\rangle=$ $=\exp \{-i H t\}\left|\psi_{0}\right\rangle$, генерируемого гамильтонианом системы. Отклонение вектора состояний $|\psi(t)\rangle$ от геодезической линии, связывающей состояния $\left|\psi_{0}\right\rangle$ и $\left|\psi_{1}\right\rangle$, определяется через кривизну пространства. Для введения понятия кривизны рассмотрим случай эволюции из двух состояний $\left|\psi_{0}\right\rangle$ в $\left|\psi_{1}\right\rangle$. На первом этапе предположим, что осуществляется эволюция в течение отрезка времени $\Delta t$ из начального состояния $\left|\psi_{0}\right\rangle$ в промежуточное $\left|\psi^{\prime}\right\rangle$ в виде $\left|\psi^{\prime}\right\rangle=e^{-\frac{i}{\hbar} H \Delta \mathrm{t}}\left|\psi_{0}\right\rangle$ и далее в течение отрезка времени $\Delta t^{\prime}$ из состояния $\left|\psi^{\prime}\right\rangle$ в состояние

$$
\begin{aligned}
& \left|\psi_{1}\right\rangle=e^{-\frac{i}{\hbar} H \Delta t^{\prime}}\left|\psi^{\prime}\right\rangle=e^{-\frac{i}{\hbar} H\left(\Delta \mathrm{t}+\Delta t^{\prime}\right)}\left|\psi_{0}\right\rangle\left|\psi_{1}\right\rangle= \\
& =e^{-\frac{i}{\hbar} H \Delta t^{\prime}}\left|\psi^{\prime}\right\rangle=e^{-\frac{i}{\hbar} H\left(\Delta \mathrm{t}+\Delta t^{\prime}\right)}\left|\psi_{0}\right\rangle,
\end{aligned}
$$

где $H-$ не зависящий от времени гамильтониан. Без потери общности в дальнейшем принимается $\Delta t=\Delta t^{\prime}$. Отклонение квантовой эволюции от геодезической линии, связывающей состояния $\left|\psi_{0}\right\rangle$ и $\left|\psi_{1}\right\rangle$, может быть охарактеризовано максимальным значением величины $\left|\left\langle\psi^{\prime} \mid \psi(\xi)\right\rangle\right|^{2}$ параметризованной $\xi$. При $\max \left|\left\langle\psi^{\prime} \mid \psi(\xi)\right\rangle\right|^{2}=1$ состояние $\left|\psi^{\prime}\right\rangle$ принадлежит геодезической линии. Отклонение $\left|\psi^{\prime}\right\rangle$ от геодезической увеличивается с уменьшением величины $\max \left|\left\langle\psi^{\prime} \mid \psi(\xi)\right\rangle\right|^{2}$. Обычно вводится выражение

$$
1-\max \left|\left\langle\psi^{\prime} \mid \psi(\xi)\right\rangle\right|^{2}=\min \left(1-\left|\left\langle\psi^{\prime} \mid \psi(\xi)\right\rangle\right|^{2}\right),
$$
которое эквивалентно нулю, когда отклонение равно нулю, и положительно возрастает, когда отклонение увеличивается. Нетрудно заметить, что данное выражение эквивалентно метрике расстояния $F S$. 
Дополнительная точка зрения на квантовый алгоритм может быть рассмотрена с позиции траекторий в пространстве унитарных преобразований. При заданной траектории $|\psi(s)\rangle$ квантового алгоритма можно определить оператор $U$ таким образом, что выполняется соотношение $U|\psi(0)\rangle=|\psi(1)\rangle$, определить программу как траекторию в пространстве унитарных операторов $O(s)$, и при этом выполняется $O(s=0)=1$ и $O(s=1)=U$. В таком случае вычислительная сложность заданной программы определяется количеством гейтов, необходимых для определения реализации данной траектории квантового алгоритма. Рассматриваемая траектория генерируется однозначно унитарным оператором $H$, который идентифицируется как гамильтониан системы, формирующий структуру квантового алгоритма и его эволюцию вдоль траектории. Тогда можно записать $O(t)=$ $=e^{-i H t} O(0) e^{i H t}$ и аналогично для $|\psi(t)\rangle$. В случае выполнения условия $[H, O(0)] \neq 0$ возникает вопрос о том, как потенциально простой начальный оператор $O(0)$ принимает в процессе эволюции во времени сложную структуру. Для заданной траектории $O(s)$ возможно ввести определение оператора Ляпунова $L_{s}$ в виде решения уравнения Ляпунова:

$$
\frac{L_{S} O(s)+O(s) L_{s}}{2}=\frac{d O(s)}{d s} .
$$

Для найденного решения $L_{s}$ можно определить функцию квантовой информации Фишера $F(s)$ как $F(s)=\operatorname{Tr}\left[O(s) L_{S}^{2}\right]$. Естественным отношением между функцией квантовой информации Фишера и метрикой различимости состояний на гильбертовом пространстве является введенная интуитивная геометрическая интерпретация сложности. Несложно представлять длину траектории в метрике пространства унитарных операторов, которая задается в виде обратной квантовой функции Фишера. По сути данная метрика сложности имеет дуальный характер по отношению к стандартной метрике различимости, которая в силу дуальности просто является соотношением неопределенности «время-энергия». Квантовая информация Фишера зависит от вариаций поведения системы во времени, тогда как энтропия является величиной равновесной и не зависит от изменений распределений вероятностей во времени. В связи с отмеченным отношение между сложностью и энтропией с этой точки зрения отражает отношение между информацией о состоянии (энтропия) и относительным состоянием во времени (сложность).

Допустим, что рассматривается траектория оператора $O(s)$, определено однопараметрическое семейство собственного базиса $\left|\phi_{n}(s)\right\rangle$ в виде $O(s)\left|\phi_{n}(s)\right\rangle=\rho_{n}(s)\left|\phi_{n}(s)\right\rangle$.

В этом базисе имеем $O(s)=\sum_{n} \rho_{n}(s)\left|\phi_{n}(s)\right\rangle \times$ $\times\left\langle\phi_{n}(s)\right|$. Рассмотрим уравнение Ляпунова для оператора траектории $O(s)$ и разрешим уравнение для определения $L_{s}$. Тогда имеем

$$
\begin{aligned}
& L_{S}=\sum_{n}\left(\frac{\partial_{s} \rho_{n}(s)}{\rho_{n}(s)}\left|\phi_{n}(s)\right\rangle\left\langle\phi_{n}(s)\right|+\right. \\
& +2 \rho_{n}(s) \sum_{m \neq n} L_{n, m}\left|\phi_{n}(s)\right\rangle\left\langle\phi_{m}(s)\right|+ \\
& \left.+2 \rho_{n}(s) \sum_{m \neq n} L_{n, m}\left|\phi_{m}(s)\right\rangle\left\langle\phi_{n}(s)\right|\right),
\end{aligned}
$$

где $\left|\partial_{s} \phi_{n}(s)\right\rangle=\sum_{m} L_{m, n}\left|\phi_{m}(s)\right\rangle$.

Оператор Ляпунова $L_{s}$ в случае, когда задан гамильтониан эволюции $H$, определяет траекторию $O(s)=e^{-i H s} O(0) e^{i H s}$. Применяя к $O(0)$ представление в виде $O(0)=\sum_{n} \rho_{n}\left|\phi_{n}\right\rangle\left\langle\phi_{n}\right|$, как результат получаем $L_{s}=e^{i H s} L_{0} e^{i i s s}$ с учетом (см. [20]), что

$$
L_{0}=2 i \sum_{n, m} \frac{\left\langle\phi_{m}|[H, O(0)]| \phi_{n}\right\rangle}{\rho_{m}+\rho_{n}}\left|\phi_{n}\right\rangle\left\langle\phi_{m}\right| .
$$

С определением функции оператора Ляпунова $L_{s}$ определяется квантовая функция Фишера $F(s)=\operatorname{Tr}\left[O(s) L_{S}^{2}\right]$. Тогда $\operatorname{Tr}\left[O(s) L_{S}\right]=0$ и для случая временной эволюции с заданным гамильтонианом квантовая функция Фишера является постоянной. При этом при определенном через временную эволюцию гамильтониане сама квантовая функция Фишера также постоянная и определяется как $F=\operatorname{Tr}\left[O(0) L_{0}^{2}\right]$

Из изложенного следует, что при построении математической модели необходимо проверять ее корректность, устойчивость и условия термодинамического критерия физической реализуемости: нарушение хотя бы одного из этих условий ведет, по существу, к нарушению остальных условий. При этом необходимо, чтобы условия корректности и устойчивости исследуемой математической модели совпадали с соответствующим понятием их устойчивости. Этот вывод является обоснованием часто встречающейся в системной инженерии интуитивной замены понятия корректности обыкновенных дифференциальных уравнений на понятие их устойчивости.

Следовательно, выбор физического базиса проведения эксперимента и определение ма- 
тематического способа (задания критерия корректного описания) модели ОУ существенно влияют на качество интерпретации результата процесса обработки экспериментальных данных и извлечения объективных знаний из динамического поведения самого ОУ.

\section{Информационно-термодинамические условия и ограничения на возможность совершения полезной работы на основе извлеченной информации}

Современная физика (феноменологическая термодинамика) утверждает, что необходимо совершить полезную работу для извлечения количества информации о поведении исследуемой системы, затратив при этом дополнительный энергетический ресурс и вместе с этим потеряв полезную работу за счет возрастания энтропии в поведении исследуемого объекта и системы измерения извлекаемой информации - физический закон интеллектуальной ИТ образовательного процесса. В этом случае справедливы [21] следующие информационно-термодинамические ограничения:

$$
W_{e x t}^{S} \leq-\Delta F^{S}+k_{B} T I, W_{\cos t}^{S} \geq k_{B} T I,
$$

где $W_{\text {ext }}^{S}-$ количество извлекаемой из системы $S$ работы (не путать с обозначением термодинамической энтропии $S$ ), определяемое количеством (квантово-классической взаимной) информации $I$, которая является мерой точности измерений и знаний о системе $S$ и ограничено сверху; $W_{\text {cost }}^{S}-$ полная стоимость измерения и считывания информации и ограничено снизу; $\Delta F^{S}-$ свободная энергия системы $S ; k_{B} T I$ ( $T$ - температура и $k_{B}$ - постоянная Больцмана) - количество работы, которое можно извлечь из термодинамического цикла и затратить на считывание одного бита информации. Таким образом, согласно (17) существуют объективные границы на количество извлекаемых знаний из измерений поведения исследуемой системы, а знания позволяют совершать полезную работу.

Пример. Информационно-термодинамические оценки затрат ресурса на извлечение количества знаний из проиессов измерения. В микросистемах термодинамические величины, такие как работа, тепло и внутренняя энергия, не являются постоянными величинами и флуктуируют. Случайные флуктуации могут нарушать второй закон термодинамики, но в среднем второй закон термодинамики на макроуровне выполняется, если начальное состояние системы находится в тепловом равновесии, то есть $\langle\Delta F-W\rangle \leq 0$, где, как и ранее, $\Delta F-$ свободная энергия как мера различия между состояниями системы; $W$ - совершаемая работа; знак 〈 > означает усреднение по ансамблю множества состояний системы. Однако управление с обратной связью позволяет целенаправленно выборочно манипулировать только флуктуациями так, что выполняется строгое неравенство $\Delta F-W>0$. Данное выражение эквивалентно использованию информации о поведении системы [22] - прототип демона Максвелла. Термин «обратная связь» означает, что выбор стратегии управления зависит от результатов измерений на выходе системы управления. Другими словами, метод управления с обратной связью эквивалентен применению систем управления с замкнутым контуром. Управление с обратной связью позволяет использовать информацию в качестве ресурса в виде свободной энергии системы [23]. Отметим, что Сциллард разработал модель преобразования одного бита информации в свободную энергию (или работу) в количестве $k_{B} T \ln 2$. Таким образом, второй закон термодинамики обобщается в виде $\langle\Delta F-W\rangle \leq k_{B} T I$. Здесь $I$ означает количество взаимной информации, извлеченной измерением состояния системы, то есть является объективной величиной [24, 25].

Следовательно, демон Максвелла (по типу Сцилларда) - это активный интеллектуальный агент, который способен оценить вход (используемое содержание информации) и выход (приобретенную энергию) при управлении с обратной связью, реализует идею преобразования информации в энергию и является информационно-термодинамическим решением обратной проблемы оценки потерь на извлечение информации о свойствах модели ОУ.

Благодаря синергетическому эффекту создается дополнительный информационный ресурс и мультиагентная система способна решать сложные динамические задачи для выполнения командной работы. Данная задача не может быть выполнена каждым элементом (агентом) системы отдельно в различных средах без внешнего управления, контроля или координации, однако обмен знаниями и информацией позволяет выпол- 
нять полезную командную работу для достижения цели управления в условиях неопределенности исходной информации и ограничений на возможное потребление полезных ресурсов.

В частности (см. пример ниже), для систем управления с обратной связью объем извлекаемой полезной работы удовлетворяет неравенству

$$
W_{\max }(t)=k \int_{0}^{t} T_{\min } \dot{I}_{c} d t^{\prime} \leq k T I_{c},
$$

где $k$ - это постоянная Больцмана; $T_{\min }(t)$ интерпретируется системой как самая низкая достижимая температура во времени $t$ для контроля обратной связи, предполагая $T(0)_{\min } ; I_{c}$ определяет количество информации Шеннона (перенос энтропии), извлеченное системой из процесса измерения.

Пример. Информационно-термодинамический анализ интеллектуальной когнитивной системы управления. Рассмотрим струк- турную схему (рис. 2) гибридной когнитивной ИСУ. Ее особенностью является наличие двух взаимодействующих регуляторов - нечеткого интеллектуального и когнитивного. Отметим, что БЗ интеллектуального технического регулятора и БЗ когнитивного регулятора создаются на основе единой программно-алгоритмической платформы - оптимизаторе Б3 на мягких вычислениях, используя сигнал ошибки управления и соответствующие обучающие сигналы, описывающие динамическое поведение ОУ или электроэнцефалограмму коры головного мозга человекаоператора.

Ошибка управления содержит информацию о непредвиденной ситуации управления и поступает на входы рассматриваемых регуляторов. Выходные сигналы регуляторов отражают реакции Б3, которые в общем случае являются неполными и могут приводить к потере робастности системы управления. Оба

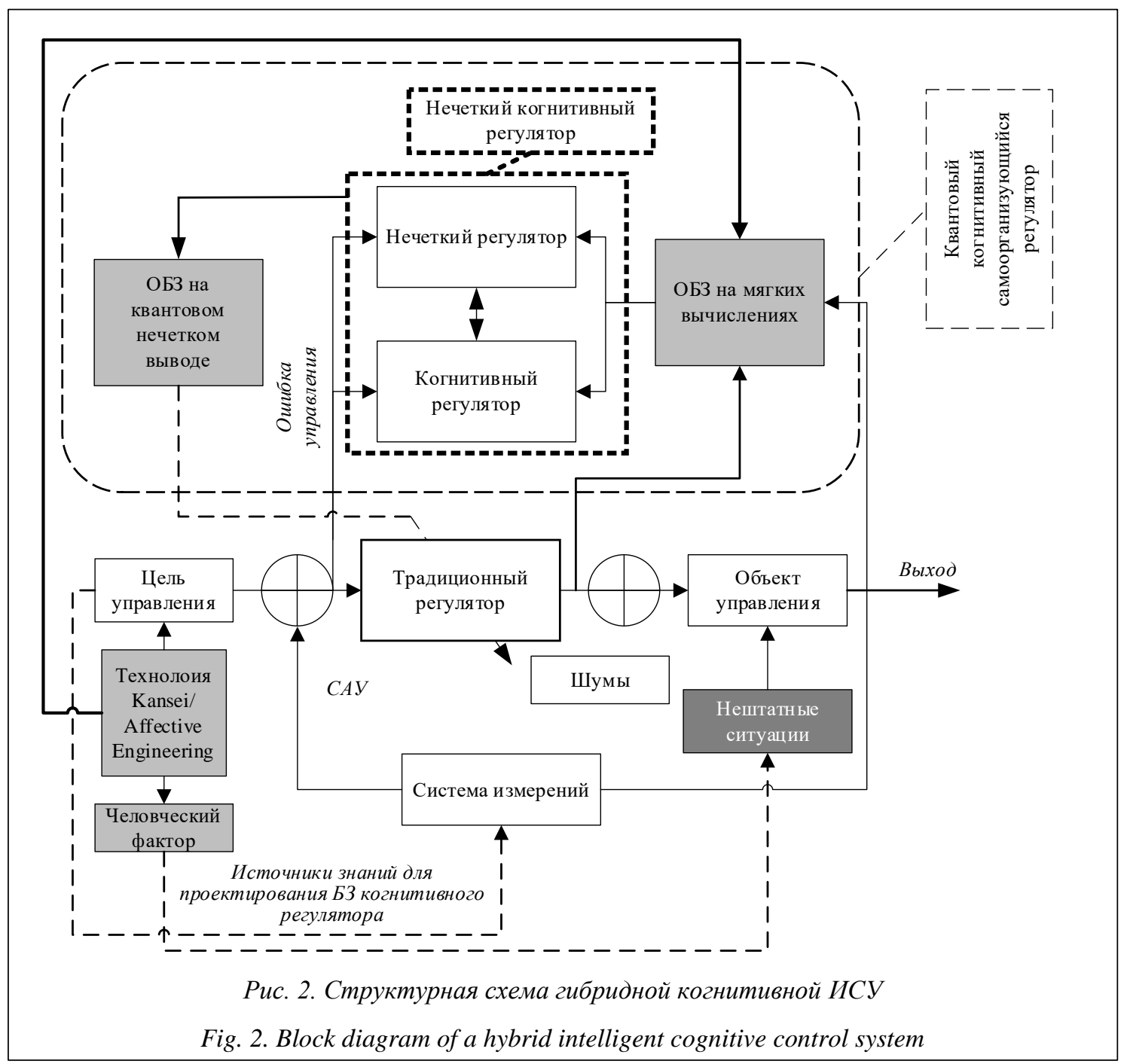


сигнала поступают в блок квантового нечеткого вывода, осуществляющий самоорганизацию неполных Б3, формируя в реальном времени новую робастную БЗ гибридного квантового интеллектуального когнитивного регулятора.

Обобщением уравнений (3) и (4) является следующая система уравнений:

$$
\begin{aligned}
& \underbrace{\frac{d V}{d t}}_{\text {устойчивость }}=\underbrace{\sum_{i=1}^{n} q_{i} \varphi\left(q, t, k\left(S_{c o}-\left(S_{T c}+S_{C c}\right)\right), u\right)}_{\text {управляемость }}+ \\
& +\underbrace{\left(S_{c o}-\left(S_{T c}+S_{C c}\right)\right)\left(\dot{S}_{c o}-\left(\dot{S}_{T c}+\dot{S}_{C c}\right)\right)}_{\text {робастность }} \leq 0, \\
& \underbrace{\frac{d V}{d t}}_{\text {устойчивость }}=\underbrace{\sum_{i=1}^{n} q_{i} \varphi\left(q, t, k\left(H_{c o}-\left(H_{T c}+H_{C c}\right)\right), u\right)}_{\text {управляемость }}+ \\
& +\underbrace{\left(H_{c o}-\right.}_{\text {робастность }}\left(H_{T c}^{\left.\left.H_{T c}+H_{C c}\right)\right)\left(\dot{H}_{c o}-\left(\dot{H}_{T c}+\dot{H}_{C c}\right)\right)} \leq 0,\right.
\end{aligned}
$$

где $\left(S_{T c}+S_{C c}\right)$ и $\left(H_{T c}+H_{C c}\right)$ означают совместные термодинамические и информационные энтропии технического интеллектуального и когнитивного регуляторов соответственно.

Обсудим особенности соотношения (18) применительно к системе уравнений для интеллектуального когнитивного управления (19) и (20).

Известное выражение второго закона феноменологической термодинамики утверждает, что максимальное (усредненное) количество работы, извлекаемое из системы при взаимодействии с простым тепловым резервуаром, не может превышать количества свободной энергии и проявляет стремление к понижению при переходе системы из начального в конечное состояние. Однако, как следовало из анализа поведения теплового двигателя (тепловой машины) Сцилларда, возможно нарушение такого ограничения при допущении предположения о доступности дополнительной информации интеллектуальному агенту, извлекающему на ее основе работу из тепловой машины (открытая система с информационным обменом). Для учета такой возможности второй закон феноменологической термодинамики обобщается на основе введения наблюдателя (демона) с контуром управления обратной информационной связью. В частности, показано, что при применении управления с обратной информационной связью количество извлекаемой работы $W$ должно удовлетворять неравенству типа (17) следующего вида:

$$
W \leq k I T_{c},
$$

где $k$ - постоянная Больцмана; $T$ - температура теплового резервуара; $I_{c}$ определяет так называемый перенос энтропии к регулятору от системы измерения [26, 27]. При этом предполагается, что при переходе от начального к конечному состоянию снижения свободной энергии не происходит. Имеется также возможность разработать протоколы обратных связей, позволяющие сформировать уравнения определения $W$, применяя обратимые и квазистатичные преобразования. В первом приближении данного подхода определяется составляющая конечного времени для определяющего $W$ уравнения, характеризующая максимальную работу, которую можно извлечь с применением управления с обратной связью, в терминах переноса энтропии. Результат описывается на основе исследования поведения существенно демпфированной системы на основе модели решения уравнения Ланжевена. Максимальное количество извлекаемой работы за период $t$, $W_{\max }(t)$, определяется [28] через интеграл вида

$$
W_{\max }(t)=k \int_{0}^{t} T_{\min } \dot{I}_{c} d t^{\prime} \leq k T I_{c}(t) .
$$

Здесь $T_{\min }(t) \quad$ интерпретируется как наименьшая температура, достигаемая системой за время $t$ непрерывного управления с обратной связью при предположении, что начальное значение равновесия системы $T_{\min }(0)=T$. Так как $T_{\min }(t) \leq T$, верхняя граница в (22) получается из применения (21).

Количество переноса энтропии $I_{c}(t)$ определяется через количество передаваемой информации регулятору от системы измерений в интервале $[0, t]$. Поэтому оптимально применяемое количество переноса энтропии регулятором позволяет извлечь полезную (от $k T_{\min } \ln 2$ до $\left.k T \ln 2\right)$ работу.

Кроме того, для широкого класса динамических систем получено выражение для определения количества переноса энтропии $I_{c}(t)$, применимое для случая как непрерывного, так и дискретного времени. В частности, новое соотношение получено в замкнутой математической форме для определения количества переноса энтропии (вне зависимости от закона и применяемого вида обратной связи). Для линейных систем размерности, превышающей единицу, то есть для динамических систем с квадратичным гамильтонианом и контактом с тепловым резервуаром, показано выполнение соотношения

$$
W_{\max }(t) \simeq k \int_{0}^{t} T_{\min } \dot{I}_{c} d t^{\prime}
$$


и выполняется равенство асимптотически $t \rightarrow \infty$, то есть система находится в неравновесном состоянии. Более того, для всех состояний выполняются неравенство $W \leq k T I_{c}(t)$ и второй закон для конечных интервалов времени. Квадратичная форма гамильтониана является типовой для систем с тепловыми флуктуациями при комнатной температуре, находящихся в состоянии с минимальной энергией. Теория оптимального управления дает возможность вычислить $T_{\min }(t)$ и определить характер закона обратной связи, например, для линейных систем по принципу разделения переменных, наблюдения параметров траекторий и управления. Оценка измерения траектории может быть дана эффективно на основе применения фильтра Калмана-Бьюси, который позволяет максимально эффективно использовать информацию, содержащуюся в измерениях на основе минимума вариаций оценки ошибки.

Пример. Рассмотрим систему, схема которой представлена на рисунке 3 , состоящую из электрической емкости $C$, сопротивления $R$ с тепловым шумом (тепловой резервуар) и регулятора обратной связи (демона) с возможным доступом к измерению показаний напряжения вольтметра.

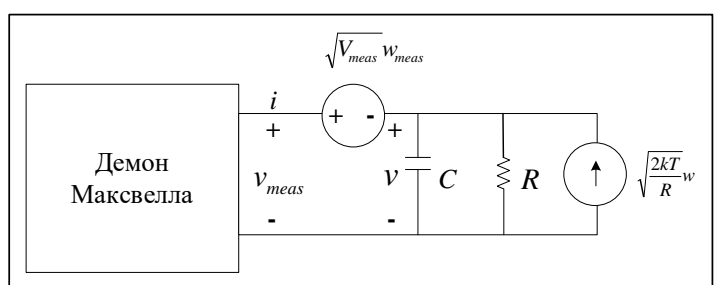

Рис. 3. Демон (регулятор с обратной связью), подключенный к конденсатору с температурой Т и источнику шума напряжения интенсивностью $V_{\text {mеаs }}$

Fig. 3. The daemon (closed-loop controller) connected to a capacitor with a temperature $T$ and a voltage noise source with $V_{\text {meas }}$ intensity

Демон имеет возможность наблюдать величину тока $i$ и имеет доступ к измерению шума напряжения $\vartheta_{\text {meas }}$. Резистор содержит случайный шум, описываемый моделью Джонсона-Найквиста. Схема на рисунке 3 моделируется на основе существенно демпфированного уравнения Ланжевена следующего вида:

$$
\begin{aligned}
& \tau \dot{\vartheta}=-\vartheta+R i+\sqrt{2 k T R} w, \vartheta(0)=0 \\
& \vartheta_{\text {meas }}=\vartheta+\sqrt{V_{\text {meas }}} w_{\text {meas }}, \vartheta(0)^{2}=\frac{k T}{C},
\end{aligned}
$$

где $9(0)$ - гауссовский шум; $w$ и $w_{\text {meas }}-$ некоррелированные гауссовские белые шумы $\left.\left.\left.\left(\left\langle w(t) w\left(t^{\prime}\right)\right\rangle\right)=\left\langle w_{\text {meas }}(t) w_{\text {meas }}\left(t^{\prime}\right)\right)\right\rangle=\delta\left(t-t^{\prime}\right)\right)\right)$ $V_{\text {meas }}$ - интенсивность измеримого шума; $\tau=R C-$ постоянная величина открытой системы. Шум измерения $\sqrt{V_{\text {meas }}} w_{\text {meas }}$ можно рассматривать как шум Джонсона-Найквиста в канале связи между конденсатором и демоном, сопротивление которого для простоты заложено в демоне. Поток тепла к сопротивлению определяется как $\dot{Q}$, скорость извлекаемой демоном работы задается в виде $\dot{W}$, и они подчиняются первому закону термодинамики: $\dot{U}=\dot{Q}-\dot{W}$, где $U=\frac{1}{2} C\left(\vartheta^{2}\right) \equiv \frac{1}{2} k T_{C}$;

$$
\dot{Q}=\frac{k}{\tau}\left(T-T_{C}\right) ; \dot{W}=-\langle\vartheta i\rangle .
$$

Эффективная текущая температура (кинетическая температура) емкости обозначается как $T_{C}$, а внутренняя энергия - $U$. Напряжение $\vartheta_{\text {meas }}$ является измеримой величиной и «питает» демона информацией: физически означает измерение флуктуирующего шума напряжения в емкости $\vartheta$ демоном.

Данная задача оптимизации решается в режиме (без информации о траекторной реализации $\left.\left(\vartheta_{\text {meas }}\right)_{0}^{t}\right)$ на основе метода динамического программирования. Состояние фильтра Калмана-Бьюси обозначается как $\hat{\vartheta}$ и рассматривается как решение уравнения

$$
\tau \frac{d}{d t} \hat{\vartheta}=-\hat{\vartheta}+R i+K\left(\vartheta_{\text {meas }}-\hat{\vartheta}\right), \hat{\vartheta}(0)=0,
$$

где $K$ является функцией времени и подлежит определению. Для фиксированных уровней шума существует оптимальное значение коэффициента усиления $(K)_{0}^{t}$, называемое коэффициентом усиления Калмана, при котором достигается минимальная оценка значения вариации ошибки $\min _{(\kappa)_{0}^{t}}[\vartheta(t)-\hat{\vartheta}(t)]^{2}$.

В результате применение методологии динамического программирования приводит к исследованию уравнения фильтра Риккати, которое для рассматриваемой задачи записывается в следующем виде:

$$
\tau \dot{T}_{\min }=2\left(T-T_{\text {min }}\right)-\frac{\sigma T_{\min }^{2}}{2 T}, T_{\min }(0)=T,
$$

где оптимальный коэффициент усиления $K$ выражен параметризацией через решение $\left(T_{\min }\right)_{0}^{t}, K=\frac{\sigma T_{\min }}{2 T}, \sigma \equiv \frac{2 k T R}{V_{\text {meas }}}$ и $\sigma$ характеризует 
фундаментальное природное поведение теплового резервуара в виде измеримой интенсивности случайного теплового шума.

Для получения оценки максимальной работы в (23) необходимо иметь оценку информационного потока по непрерывному каналу обратной связи неопределенной части измеримого напряжения $\vartheta$ в виде измеримой величины $\vartheta_{\text {meas }}$. Такой величиной является перенос энтропии [28]. Оценка непрерывного по времени предела переноса энтропии имеет следующий вид:

$$
I_{C}(t)=I\left(\left(\vartheta(0),(w)_{0}^{t}\right) ;\left(\vartheta_{\text {meas }}\right)_{0}^{t}\right) .
$$

Такая величина называется количеством взаимной информации между неопределенным начальным значением напряжения $\vartheta(0)$ и случайными значениями траектории теплового шума $w$, возникающего при измерении траектории $\vartheta_{\text {meas. }}$ К Количество взаимной информации двух случайных величин $\xi$ и $\theta$ определяется в виде

$$
I(\theta ; \xi)=\int \ln \left(\frac{d P_{\theta \xi}}{d\left(P_{\theta} \otimes P_{\xi}\right)}\right) d P_{\theta \xi} \geq 0
$$

и эквивалентно количеству (дифференциальной) энтропии Шеннона случайной величины $\xi$, убывающей при наличии знаний о случайной величине $\theta$, и наоборот. Здесь $P_{\theta \xi}, P_{\theta}$ и $P_{\xi}$ описывают взаимные и маргинальные меры распределения вероятностей случайных величин $\theta$ и $\xi$. Тогда для данной задачи количественно перенос энтропии определяется следующим выражением:

$$
\begin{aligned}
& I_{c}(t)=\frac{1}{2 V_{\text {meas }}} \int_{0}^{t}\left\langle[\vartheta-\hat{\vartheta}]^{2}\right\rangle d t^{\prime}= \\
& =\frac{1}{2 V_{\text {meas }}} \int_{0}^{t} \frac{k T_{\text {min }}}{C} d t^{\prime}=\frac{\sigma}{4 \tau} \int_{0}^{t} \frac{T_{\min }}{T} d t^{\prime}, \tau=R C .
\end{aligned}
$$

Отметим, что $I_{c}$ не зависит от описания природы демона, например, деталей описания коэффициента его усиления обратной связи $G$. Сам демон может оптимально управлять извлечением работы (23) с помощью использования измерений $\vartheta_{\text {meas }}$ и выбора инжекции тока $i$. На интуитивном уровне интерпретации демон может воспроизводить положительную работу со скоростью $\dot{W}$, если будет принимать значения тока как $i<0$ при корректной оценке $\vartheta>0$, и наоборот.

Модель демона традиционно рассматривают как слабо наблюдаемую систему с малыми флуктуациями, при действии на которую энергия извлекается в противоречии второму закону термодинамики. В более со- временной интерпретации демон рассматривается как регулятор, содержащий систему измерения (цифровой или аналоговый вычислитель), способный определить наиболее оптимальное действие для реализации исполнительным устройством. Извлеченная информация должна храниться в памяти компьютера в период необходимого использования. Демон Ландауэра-Пенроуза-Беннета рассматривает память компьютера совместно с принципом Ландауэра, согласно которому для стирания одного бита при температуре $T$ необходимо затратить на преодоление диссипативных сил теплового резервуара работу как минимум $k T \ln 2$ как ключевой позиции для восстановления выполнения условий второго закона термодинамики.

Если наблюдателю в виде демона Максвелла доступны микроскопические степени свободы, то второй закон термодинамики может быть нарушен. Сциллард показал из анализа модели демона Максвелла, что из термодинамического цикла извлекается работа в виде $k T \ln 2$. Более того, извлекаемая из системы работа $W_{\text {ext }}^{S}$ определяется количеством информации (или квантово-классической взаимной информацией) $I$, которая измеряет знания о системе при измерении. Одновременно подобное соотношение в виде нижней границы существует для полной стоимости $W_{\cos t}^{M}$ измерения и стирания информации $W_{e x t}^{S} \leq-\Delta F^{S}+k T I$ и $W_{\text {cost }}^{M} \geq k T I$, где $\Delta F^{S}$ определяет свободную энергию системы. Тогда нетрудно заметить, что скорость извлекаемой работы $\dot{W}_{\text {ext }}$ ограничена величиной $\dot{W}_{e x t} \leq k T \dot{I}$, то есть скоростью извлекаемой информации.

Таким образом, регулятор имеет возможность совершить полезную работу за счет извлеченной информации с ограничениями в виде (22). На рисунке 4 приведено интегральное описание информационно-термодинамического закона распределения качеств управления, применяемого в задачах проектирования когнитивных ИСУ.

\section{Заключение}

Отметим некоторые дополнительные выводы из анализа особенностей информационно-термодинамического закона квантовой самоорганизации когнитивных робастных ИСУ на рисунке 4 с позиции теории динамических робототехнических систем. 
Информационно-термодинамические аспекты когнитивного интеллектуального управления:

устойчивость, управляемость и робастность интеллектуального когнитивного управления

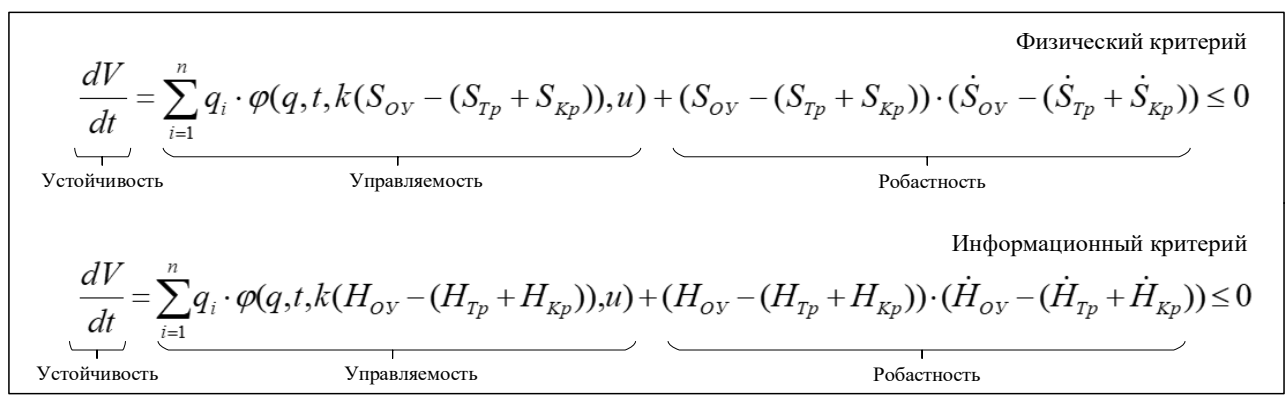

Извлеченная квантовая информация, скрытая в классических состояниях, является

информационным ресурсом для совершения дополнительной полезной работы

$$
\langle\Delta S\rangle+k_{B} \ln 2 \Delta H \geq 0 \quad \text { Модифицированный второй закон термодинамики }
$$

$$
W_{\max }(t)=k \int_{0}^{t} T_{\min } I_{c} d t^{\prime} \leq k T I_{c} \quad \begin{aligned}
& \text { Информационный закон извлечения полезной } \\
& \text { максимальной работы }
\end{aligned}
$$

Рис. 4. Информационно-термодинамический закон распределения качеств управления

Fig. 4. The information-thermodynamic law of control quality distribution

Физический (термодинамический) критерий (19) самоорганизации робастных ИСУ показывает, что уровень робастности возможно повысить за счет управления производством энтропии технического регулятора, который позволяет снизить потери полезного ресурса объекта управления. Информационный критерий (20) показывает, что негэнтропия когнитивного регулятора снижает требования к количеству необходимой начальной информации для гарантированного достижения проектируемого уровня робастности. Таким образом, извлекаемая из Б3 когнитивного регулятора информация предоставляет возможность получить дополнительный ресурс для совершения полезной работы, что эквивалентно возможности целенаправленного действия на объект управления для гарантированного достижения цели управления. Этот факт подтверждает справедливость утверждения о физической природе информации [29-32], и целенаправленное управление, использующее данную информацию, возможно за счет совершения извлеченной на ее основе дополнительной полезной работы.

Приведенные на рисунке 4 соотношения характерны для диссипативных объектов управления с высокоэнергетическими уров- нями, а также для применяемых в данных объектах регуляторов с обратной связью без потерь с низким энергетическим уровнем. В этом общем случае энергия стремится к передаче управления от объекта регулятору, тем самым энергия объекта управления убывает, энергия регулятора увеличивается и энергия эмуляции (но не физическая энергия) аккумулируется регулятором. И наоборот, если применяемый регулятор с высоким энергетическим уровнем, то энергия может передаваться от регулятора объекту управления, так как регулятор может воспроизводить реально физическую энергию для управления достижимостью требуемого энергетического потока.

Тогда, если состояния регулятора совпадают с высоким уровнем энергии эмуляции, возможно восстановить данные состояния, удаляя энергию эмуляции так, что энергия эмуляции не возвращается к объекту управления. В этом случае общая замкнутая система состоит из объекта управления и регулятора и обладает прерывистыми потоками, такими, что возможно организовать логическое переключение в сочетании с непрерывной динамикой и сформировать модель описания на основе импульсных дифференциальных уравнений [29]. 
Основная цель робастного интеллектуального когнитивного управления заключается в поддержке оптимального соотношения в распределении качеств управления, таких как устойчивость, управляемость и робастность, при выполнении термодинамических и информационных соотношений (рис. 4) и выполнении роли информационно-термодинамического компенсатора стабилизации процессов управления. Множество обнуления определяется как множество всех точек в пространстве состояний регуляторов с замкнутыми обратными связями, которому соответствуют регуляторы с убывающей энергией эмуляции. При обнулении состояний регулятора энергия объекта управления никогда не может возрастать после первого же события обнуления состояний.

Более того, если энергия замкнутой системы управления сохраняется между событиями обнуления состояний регулятора, убывание энергии объекта управления сопровождается соответствующим возрастанием энергии эмуляции. Таким образом, поддержка оптимального информационно-термодинамического распределения между устойчивостью, управляемостью и робастностью за счет процессов самоорганизации знаний может быть реализована при помощи нового квантового алгоритма управления самоорганизацией знаний в виде неточных Б3 на основе операций квантовых вычислений, которые отсутствуют в технологиях мягких вычислений.

Приведенные соотношения между количеством информации, извлекаемой свободной энергии и работы подтверждают отмеченный выше вывод: робастность ИСУ возможно повысить за счет производства энтропии когнитивного регулятора, который уменьшает потери полезного ресурса объекта управления, а негэнтропия когнитивного регулятора снижает требования к минимуму исходной информации для достижения робастности.

Поэтому извлекаемая информация, основанная на знаниях в Б3 когнитивного регулятора, позволяет получить дополнительный ресурс для совершения полезной работы, что эквивалентно появлению целенаправленного действия на объект управления для гарантированного достижения цели управления.

\section{Лuтература}

1. Петров Б.Н., Уланов Г.М., Гольденблат И.И., Ульянов С.В. Теория моделей в процессах управления: Термодинамические и информационные аспекты. М.: Наука, 1978. 224 с.

2. Ulyanov S.V. Self-Organized Intelligent Control System. US Patent no. 6,411,944B1.2002.

3. Marcolli M. Motivic information. Bollettino dell'Unione Matematica Italiana, 2019, no. 12, pp. 19-41. DOI: 10.1007/s40574-018-0167-z.

4. Combe C.N., Manin Yu.I., Marcolli M. Geometry of information: classical and quantum aspects. ArXiv, pp. 1-44. URL: http://www.its.caltech.edu/ matilde/GeometryInformationFmflds.pdf (дата обращения: 1.08.2021).

5. Стратонович Р. Теория информации. М.: Советское радио, 1975. 424 с.

6. Yufik Y. The understanding capacity and information dynamics in the human brain. Entropy, 2019, vol. 21, no. 3, pp. 308. DOI: 10.3390/e21030308.

7. Brandao F.G.S.L., Horodecki M., Ng N.H.Y., Oppenheim J., Wehner S. The second laws of quantum thermodynamics. PNAS, 2013, vol. 112, no. 11, pp. 3275-3279. DOI: 10.1073/pnas.1411728112.

8. Sagawa T. Thermodynamic and logical reversibilities revisited. J. of Statistical Mechanics: Theory and Experiment, 2014, vol. 2014, no. 3, pp. 3-25. DOI: 10.1088/1742-5468/2014/03/P03025.

9. Yamano T. Phase space gradient of dissipated work and information: A role of relative Fisher information. J. of Mathematical Physics, 2013, vol. 54, no. 11, art. 113301. DOI: 10.1063/1.4828855.

10. Ilgin I., Yang I.-S. Energy carries information. Int. J. of Modern Physics A, 2014, vol. 29, no. 20, art. 1450115. DOI: 10.1142/S0217751X14501152.

11. Horowitz J.M., Esposito M. Thermodynamics with continuous information flow. Physical Review X, 2014, vol. 4, art. 031015. DOI: 10.1103/PhysRevX.4.031015.

12. Renes J.M. Work cost of thermal operations in quantum and Nano thermodynamics. ArXiv, 2014. URL: https://arxiv.org/pdf/1402.3496.pdf (дата обращения: 1.08.2021).

13. Lang A.H., Fisher C.K., Mora T., Mehta P. Thermodynamics of statistical inference by cells. Physical Review Letters, 2014, vol. 113, art. 148103. DOI: 10.1103/PhysRevLett.113.148103.

14. Horowitz J.M., Sagawa T. Equivalent definitions of the quantum nonadiabatic entropy production. J. of Statistical Physics, 2014, vol. 156, no. 1, pp. 55-65. DOI: 10.1007/s10955-014-0991-1. 
15. Apollaro T.J.G., Francica G., Paternostro M., Campisi M. Work statistics, irreversible heat and correlations build-up in joining two spin chains. Physica Scripta, 2015, vol. 2015, no. 165, pp. 14-23. DOI: 10.1088/0031-8949/2015/T165/014023.

16. Hemmo M., Shenker O. Entropy and computation: The Landauer-Bennett thesis reexamined. Entropy, 2013, vol. 15, no. 8, pp. 3297-3311. DOI: 10.3390/e15083297.

17. Ульянов С.В., Бархатова И., Албу В. Квантовая релятивистская информатика: логика физических противоречий корректности и строгости математических моделей квантовых релятивистских объектов. Berlin: LAP Lambert Academic Publishing, 2015. 400 с.

18. Dodonov V.V., Manko O.V., Manko V.I., Wuensche A. Energy-sensitive and "Classical-like" distances between quantum states. Physica Scripta, 1998, vol. 59, no. 2, pp. 81-89. DOI: 10.1238/Physica.Regular.059a00081.

19. Filippov S.N., Manko V.I. Distances between quantum states in the tomographic-probability representation. Physica Scripta, 2010, no. T140, art. 014043. DOI: 10.1088/0031-8949/2010/T140/014043.

20. Gómez C. Complexity and time. Physical Review D, 2020, vol. 101, art. 065016. DOI: 10.1103/PhysRevD.101.065016.

21. Funo K., Watanabe Y., Ueda M. Thermodynamic work gain from entanglement. Physical Review A, 2013, vol. 88, art. 052319. DOI: 10.1103/PhysRevA.88.052319.

22. Toyabe S., Sagawa T., Ueda M., Muneyuki E., Sano M. Experimental demonstration of informationto-energy conversion and validation of the generalized Jarzynski equality. Nature Physics, 2010, vol. 6, no. 12, pp. 988-992. DOI: 10.1038/nphys1821.

23. Van der Meer R., Ng N.H.Y., Wehner S. Smoothed generalized free energies for thermodynamics. Physical Review A, 2017, vol. 96, art. 062135. DOI: 10.1103/PhysRevA.96.062135.

24. Tribus M., Shannon P.T., Evans R.B. Why thermodynamics is a logical consequence of information theory. AIChE J., 1966, vol. 12, no. 2, pp. 244-248. DOI: 10.1002/aic.690120208.

25. Bais F.A., Farmer J.D. The physics of information. Philosophy of Information, 2008, pp. 609-683. DOI: 10.1016/B978-0-444-51726-5.50020-0.

26. Sagawa T., Ueda M. Erratum: Minimal energy cost for thermodynamic information processing: Measurement and information erasure [Phys. Rev. Lett. 102, 250602 (2009)]. Physical Review Letters, 2011, vol. 106, art. 189901. DOI: 10.1103/PhysRevLett.106.189901.

27. Horowitz J., Sandberg H. Second-law-like inequalities with information and their interpretations. New J. of Physics, 2014, vol. 16, art. 125007. DOI: 10.1088/1367-2630/16/12/125007.

28. Sandberg H., Delvenne J.-C., Newton N., Mitter S. Maximum work extraction and implementation costs for nonequilibrium Maxwell's demons. Physical Review E, 2014, vol. 90, art. 042119. DOI: 10.1103/PhysRevE.90.042119.

29. Haddad W.M., Chellaboina V.S., Nersesov S.G. Thermodynamics: A dynamical systems approach. USA, New Jersey, Princeton: Princeton University Press, 2005, 200 p.

30. Feng Q., W. Li. Hypoelliptic entropy dissipation for stochastic differential equations. ArXiv, 2021, art. 00544. URL: https://arxiv.org/abs/2102.00544 (дата обращения: 1.08.2021).

31. Ito S., Dechant A. Stochastic time-evolution, information geometry and the Cramer-Rao Bound. Physical Review X, 2018, vol. 10, art. 021056. DOI: 10.1103/PhysRevX.10.021056.

32. Yoshimura K., Ito S. Information geometric inequalities of chemical thermodynamics. Physical Review Research, 2020, vol. 3, art. 013175. DOI: 10.1103/PhysRevResearch.3.013175.

Software \& Systems

DOI: $10.15827 / 0236-235 X .136 .524-542$
Received 17.08.21, Revised 22.09.21 2021, vol. 34, no. 4, pp. 524-542

\section{Thermodynamic constraints and information conditions of intelligent cognitive control stability, controllability, and robustness}

S.V.Ulyanov ${ }^{1,2}$, Dr.Sc. (Physics and Mathematics), Professor, ulyanovsv@mail.ru

A.A. Shevchenko ${ }^{1}$, Postgraduate Student, allabard@yandex.ru

A.V.Shevchenko ${ }^{1}$, Postgraduate Student, sh3vchenkoav@yandex.ru

O.Yu. Tyatyushkina ${ }^{1}$, Ph.D. (Engineering), Associate Professor, tyatyushkina@mail.ru

\footnotetext{
${ }^{1}$ Dubna State University - Institute of System Analysis and Control, Dubna, 141980, Russian Federation

2 Joint Institute for Nuclear Research - Laboratory of Information Technology,

Dubna, 141980, Russian Federation
} 
Abstract. The paper considers information and physical (entropy and energy) patterns, as well as the features of the model of a quantum strong artificial computational intelligence as a self-organizing intelligent control system. The model is based on the principles of minimal information entropy (in the "intelligent" space state of control signals) and the minimal generalized thermodynamic measure of the entropy production in the unified system "control object + intelligent cognitive controller". The main result of applying the self-organization process is the guaranteed possibility of achieving the necessary reliability and flexibility level of the reproducible structure of the cognitive intelligent control system.

The paper briefly describes the main physical principles of management processes allowing establishing the relationship between the qualitative characteristics of the dynamic behavior of the control object and the executive device of the automatic control system: control stability, controllability, and robustness. To achieve this purpose, it uses the information and thermodynamic approaches that combine dynamic stability (Lyapunov function), controllability and robustness criteria by a homogeneous condition.

The authors give the relations between the amount of pure work, information and the extracted free energy, which confirm the possibility of increasing the intellectual control system robustness due to the production of entropy of a cognitive controller that reduces the loss of the useful resource of the control object. In turn, the negative entropy of cognitive control reduces the requirements for the minimum initial information to achieve robustness. Based on the retrieved information from the cognitive controller knowledge base, it is possible to obtain an additional resource for useful work, which is equivalent to a targeted action on the management object, ensuring the management goal achievement.

Keywords: quantum self-organization algorithm, imperfect knowledge base, information process thermodynamics, cognitive control system.

\section{References}

1. Petrov B.N., Ulanov G.M., Goldenblat I.I., Ulyanov S.V. Model Theory in Control Processes: Thermodynamic and Informational Aspects. Moscow, 1978, 224 p. (in Russ.).

2. Ulyanov S.V. Self-Organized Intelligent Control System. US Patent no. 6,411,944B1.2002.

3. Marcolli M. Motivic information. Bollettino dell'Unione Matematica Italiana, 2019, no. 12, pp. 19-41. DOI: 10.1007/s40574-018-0167-z.

4. Combe C.N., Manin Yu.I., Marcolli M. Geometry of information: classical and quantum aspects. ArXiv, pp. 1-44. Available at: http://www.its.caltech.edu/ matilde/GeometryInformationFmflds.pdf (accessed August 01, 2021).

5. Stratonovich R. The Information Theory. 1975, 424 p. (in Russ.).

6. Yufik Y. The understanding capacity and information dynamics in the human brain. Entropy, 2019, vol. 21, no. 3, pp. 308. DOI: 10.3390/e21030308.

7. Brandao F.G.S.L., Horodecki M., Ng N.H.Y., Oppenheim J., Wehner S. The second laws of quantum thermodynamics. PNAS, 2013, vol. 112, no. 11, pp. 3275-3279. DOI: 10.1073/pnas.1411728112.

8. Sagawa T. Thermodynamic and logical reversibilities revisited. J. of Statistical Mechanics: Theory and Experiment, 2014, vol. 2014, no. 3, pp. 3-25. DOI: 10.1088/1742-5468/2014/03/P03025.

9. Yamano T. Phase space gradient of dissipated work and information: A role of relative Fisher information. J. of Mathematical Physics, 2013, vol. 54, no. 11, art. 113301. DOI: 10.1063/1.4828855.

10. Ilgin I., Yang I.-S. Energy carries information. Int. J. of Modern Physics A, 2014, vol. 29, no. 20, art. 1450115. DOI: 10.1142/S0217751X14501152.

11. Horowitz J.M., Esposito M. Thermodynamics with continuous information flow. Physical Review $X$, 2014, vol. 4, no. 3, art. 031015. DOI: 10.1103/PhysRevX.4.031015.

12. Renes J.M. Work cost of thermal operations in quantum and Nano thermodynamics. ArXiv, 2014. Available at: https://arxiv.org/pdf/1402.3496.pdf (accessed August 01, 2021).

13. Lang A.H., Fisher C.K., Mora T., Mehta P. Thermodynamics of statistical inference by cells. Physical Review Letters, 2014, vol. 113, no. 14, art. 148103. DOI: 10.1103/PhysRevLett.113.148103.

14. Horowitz J.M., Sagawa T. Equivalent definitions of the quantum nonadiabatic entropy production. J. of Statistical Physics, 2014, vol. 156, no. 1, pp. 55-65. DOI: 10.1007/s10955-014-0991-1.

15. Apollaro T.J.G., Francica G., Paternostro M., Campisi M. Work statistics, irreversible heat and correlations build-up in joining two spin chains. Physica Scripta, 2015, vol. 2015, no. 165, pp. 14-23. DOI: 10.1088/0031-8949/2015/T165/014023.

16. Hemmo M., Shenker O. Entropy and computation: The Landauer-Bennett thesis reexamined. Entropy, 2013, vol. 15, no. 8, pp. 3297-3311. DOI: 10.3390/e15083297.

17. Ulyanov S.V., Barkhatova I., Albu V. Quantum Algorithmic Gates. Information Analysis \& Design System in MatLab. Berlin: LAP Lambert Academic Publ., 2015, 400 p. (in Russ.). 
18. Dodonov V.V., Manko O.V., Manko V.I., Wuensche A. Energy-sensitive and "Classical-like" distances between quantum states. Physica Scripta, 1998, vol. 59, no. 2, pp. 81-89. DOI: 10.1238/Physica.Regular.059a00081.

19. Filippov S.N., Manko V.I. Distances between quantum states in the tomographic-probability representation. Physica Scripta, 2010, no. T140, art. 014043. DOI: 10.1088/0031-8949/2010/T140/014043.

20. Gómez C. Complexity and time. Physical Review D, 2020, vol. 101, no. 6, art. 065016. DOI: 10.1103/PhysRevD.101.065016.

21. Funo K., Watanabe Y., Ueda M. Thermodynamic work gain from entanglement. Physical Review A, 2013, vol. 88, no. 5, art. 052319. DOI: 10.1103/PhysRevA.88.052319.

22. Toyabe S., Sagawa T., Ueda M., Muneyuki E., Sano M. Experimental demonstration of informationto-energy conversion and validation of the generalized Jarzynski equality. Nature Physics, 2010, vol. 6, no. 12, pp. 988-992. DOI: 10.1038/nphys1821.

23. Van der Meer R., Ng N.H.Y., Wehner S. Smoothed generalized free energies for thermodynamics. Physical Review A, 2017, vol. 96, no. 6, art. 2135. DOI: 10.1103/PhysRevA.96.062135.

24. Tribus M., Shannon P.T., Evans R.B. Why thermodynamics is a logical consequence of information theory. AIChE J., 1966, vol. 12, no. 2, pp. 244-248. DOI: 10.1002/aic.690120208.

25. Bais F.A., Farmer J.D. The physics of information. Philosophy of Information, 2008, pp. 609-683. DOI: 10.1016/B978-0-444-51726-5.50020-0.

26. Sagawa T., Ueda M. Erratum: Minimal energy cost for thermodynamic information processing: Measurement and information erasure [Phys. Rev. Lett. 102, 250602 (2009)]. Physical Review Letters, 2011, vol. 106, art. 189901. DOI: 10.1103/PhysRevLett.106.189901.

27. Horowitz J., Sandberg H. Second-law-like inequalities with information and their interpretations. New J. of Physics, 2014, vol. 16, art. 125007. DOI: 10.1088/1367-2630/16/12/125007.

28. Sandberg H., Delvenne J.-C., Newton N., Mitter S. Maximum work extraction and implementation costs for nonequilibrium Maxwell's demons. Physical Review E, 2014, vol. 90, art. 042119. DOI: 10.1103/PhysRevE.90.042119.

29. Haddad W.M., Chellaboina V.S., Nersesov S.G. Thermodynamics: A Dynamical Systems Approach. USA, New Jersey, Princeton: Princeton University Press, 2005, 200 p.

30. Feng Q., W. Li. Hypoelliptic entropy dissipation for stochastic differential equations. ArXiv, 2021, art. 00544. Available at: https://arxiv.org/abs/2102.00544 (accessed August 01, 2021).

31. Ito S., Dechant A. Stochastic time-evolution, information geometry and the Cramer-Rao Bound. Physical Review X, 2018, vol. 10, art. 021056. DOI: 10.1103/PhysRevX.10.021056.

32. Yoshimura K., Ito S. Information geometric inequalities of chemical thermodynamics. Physical Review Research, 2020, vol. 3, art. 013175. DOI: 10.1103/PhysRevResearch.3.013175.

\section{Для цитирования}

УАьянов С.В., Шевченко А.А., Шевченко А.В., Тятюшкина О.Ю. Термодинамические ограничения и информационные условия устойчивости, управляемости и робастности интелмектуального когнитивного управления // Программные продукты и системы. 2021. Т. 34 . № 4. C. 524-542. DOI: 10.15827/0236-235X.136.524-542.

\section{For citation}

Ulyanov S.V., Shevchenko A.A., Shevchenko A.V., Tyatyushkina O.Yu. Thermodynamic constraints and information conditions of intelligent cognitive control stability, controllability, and robustness. Software \& Systems, 2021, vol. 34, no. 4, pp. 524-542 (in Russ.). DOI: 10.15827/0236-235X.136.524-542. 